\title{
Civilisations
}

Revue internationale d'anthropologie et de sciences

humaines

62 | 2013

Identité, culture et intimité

\section{Les stéréotypes du « macho » dans l'Etat de Jalisco}

Résistances et réinterprétations d'une figure nationaliste

Didier Machillot

\section{(2) OpenEdition}

Journals

Édition électronique

URL : http://journals.openedition.org/civilisations/3327

DOI : $10.4000 /$ civilisations.3327

ISSN : 2032-0442

Éditeur

Institut de sociologie de l'Université Libre de Bruxelles

Édition imprimée

Date de publication : 31 décembre 2013

Pagination : 165-180

ISBN : 2-87263-042-2

ISSN : 0009-8140

Référence électronique

Didier Machillot, «Les stéréotypes du «macho » dans l'Etat de Jalisco », Civilisations [En ligne],

62 | 2013, mis en ligne le 31 décembre 2016, consulté le 19 avril 2019. URL : http://

journals.openedition.org/civilisations/3327 ; DOI : 10.4000/civilisations.3327

(c) Tous droits réservés 


\title{
Les stéréotypes $d u$ " macho » dans l'Etat de Jalisco Résistances et réinterprétations d'une figure nationaliste
}

\author{
Didier MACHILLOT
}

Résumé : Si le « macho » a pendant très longtemps représenté à la fois un idéal masculin-celui de l'homme fort et viril - et national - symbole du Mexique et de sa révolution de 1910-, cette image a aussi soulevé chez d'autres, et ce dès les années 1930, l'indignation et la critique, à tel point qu'au stéréotype positif répond désormais son double négatif, le " machista", soit le "machiste " phallocrate, jugé violent et vulgaire. Devenue politique officielle, cette condamnation du " machisme " n'en soulève pas moins un certain nombre de résistances. Effectivement, outre sa dimension nostalgique et nationaliste, le " macho » incarne toujours une certaine forme d'honneur. Le stéréotype sert aussi, de manière très efficace et souvent ironique, à revendiquer son hétérosexualité contre une homosexualité stigmatisée, et ce, quitte à avoir des relations homosexuelles comme nous le découvrirons au travers de l'analyse des relations entre clients homosexuels et prostitués dits "virils ». Réemployés, réinterprétés, objets de stratégies, l'usage en contexte des stéréotypes se révèle dès lors moins " automatique » et "figé » qu'on ne le dit souvent.

Mots clefs : Mexique, stéréotype, nationalisme, machisme, masculinité.

\begin{abstract}
If the concept of "macho" has for a long time represented both a masculine ideal that of the strong and virile man - and a national one - the symbol of Mexico and its 1910 revolutionthis image has also aroused outrage and criticism in others' minds, particularly since 1930, to the point that this positive stereotype now meets direct criticism, the "machista" or the "macho" male chauvinist, and is considered violent and vulgar.

As it became an official policy, this condemnation of "machismo" also raises a number of resistant behaviors. Indeed, besides its nostalgic and nationalist dimension, the "macho" still embodies some form of honor. The stereotype also helps very effectively and often in an ironic way, to assert one's heterosexuality against a stigmatized homosexuality, and, even if it means having homosexual relationships, as we discover through the analysis of some relationships between clients and prostitutes called "manly" homosexuals.

The use of used and reinterpreted stereotypes, as subjects of strategy, in a specific context, are then less "automatic" and "rigid" than it is usually admitted.
\end{abstract}

Keywords: Mexico, stereotype, nationalism, machismo, masculinity. 


\section{Introduction}

Comme le signale Matthew Gutmann, « le Mexique en est arrivé à signifier machisme, et machisme, Mexique $»^{1}(2000: 321)$. Il est vrai que le stéréotype du " macho », né durant la révolution mexicaine et symbolisant tout d'abord le courage des combattants avant d'incarner un phallocrate (Machillot 2011), a son histoire. Si celle-ci a, de fait, été très peu étudiée malgré la popularité du « macho $»^{2}$, ce dernier a plus rarement été analysé dans les écrits académiques en tant que stéréotype et moins encore avec les méthodes et les outils de l'anthropologie, cette dernière contribuant même, parfois, comme le constate Gutmann à propos d'Oscar Lewis, à renforcer la caractérisation négative des Mexicains (2000 : 328-329).

Ser hombre de verdad en la ciudad de México. Ni macho ni mandilón (Ibid.) est, de fait, le seul ouvrage anthropologique récent à accorder une place de choix au « macho"» tout en le présentant, à la suite de pionniers comme Mendoza (1962) et Paredes (1967), comme un stéréotype. Chez Gutmann, le travail sur la masculinité et l'attention à la paternité déplacent cependant l'analyse spécifique des stéréotypes sur le «macho » pour conclure comme l'indique le titre en espagnol que le Mexicain n'est ni un « macho », ni un homme « soumis » - «ni macho ni mandilón $»^{3}$.

Qu'il éprouve le besoin de réfuter l' « être » du " macho », l'auteur s'en explique parfaitement (Gutmann 2000 : 351-353) mais c'est là aussi le défaut de son enquête : trop pressé de montrer la diversité des masculinités mexicaines, de réfuter - avec raison - l'étiquette simpliste du « macho », l'analyse des stéréotypes positifs et négatifs - le pourquoi et le comment de ces manifestations - ainsi que l'étude de l'influence que ceux-ci peuvent exercer sur le comportement des individus s'effacent quelque peu...

A la source de ce phénomène, une aporie constatée chez d'autres écrivains ou chercheurs, qui naît semble-t-il du fait d'accorder aux stéréotypes une existence réelle, consistant à les incarner littéralement dans tels ou tels acteurs ou au contraire à leur réfuter toute réalité : en d'autres termes le stéréotype est ou n'est pas. Dilemme shakespearien qui lorsqu'il ne conduit pas à une interprétation erronée éloigne semblet-il les auteurs les mieux intentionnés d'une analyse des stéréotypes en tant que tels : soit comme des catégories hypergénéralisantes et de ce fait réductrices et qui, indépendamment de leur véracité, n'en produisent pas moins des effets certains sur les individus et leurs interactions...

Suivant l'exemple de Michael Herzfeld (2007), nous nous sommes donc attaché - grâce d'abord à des recherches historiques puis, depuis 2007, à un travail sur le terrain basé sur l'observation participante et l'analyse du discours des institutions gouvernementales et de la presse - à l'analyse des stéréotypes en tant que catégories, en étudiant comment et par qui celles-ci étaient construites et utilisées en interaction, les (prises de) positions des acteurs et les usages stratégiques sous-tendant leur (ré)

1 « México llegó a significar machismo, y machismo, México » (2000:321).

2 A l'exception notable de Carlos Monsiváis (2004 : 109-124), d'une trentaine de pages chez Gutmann (2007 : 315-343) et, plus modestement, d'articles et d'une thèse de notre part (Machillot 2007, 2010 et 2011).

3 « Mandilón », forgé à partir de « mandíl», « tablier », désigne un homme « soumis » à sa femme. 
emploi. Une étude réalisée au cœur d'un Etat - Jalisco - situé à l'ouest de Mexico, plus particulièrement dans le centre de sa capitale, à Guadalajara, ainsi que dans un village de 1500 habitants de la région de Los Altos, autrement dit, dans deux lieux qui incarnent ce que les Mexicains nomment la «mexicanité »- la «mexicanidad», soit ce qui appartient en propre au Mexique.

Une réputation qui a longtemps fait de leurs habitants des « machos entre les machos » tant, historiquement, nationalisme et masculinité se confondent... Une histoire traversée par des luttes au sujet de représentations et d'imaginaires différents de la nationalité. Des affrontements idéologiques qui firent que parallèlement à l'image positive et valorisante du « macho » - celle de l'homme fort, courageux, viril, voire séducteur - surgit son double dévalorisé - l'homme vulgaire, puis dominateur.

C'est ainsi que faisant suite à un bref rappel historique de l'histoire du « macho » en tant que stéréotype nationaliste, nous verrons comment s'est imposée postérieurement et de manière très officielle l'image négative du « machiste » phallocrate. Un revirement de la part de l'Etat qui, nous le constaterons dans un deuxième point, n'est pas sans susciter au quotidien de la part des habitants de Jalisco un certain nombre de résistances voire de réappropriations et de réinterprétations genrées du stéréotype nationaliste.

\section{Le « macho " : de la revendication nationaliste au rejet du stéréotype masculin}

« Macho : Se dit d'un homme courageux ou ayant du caractère » (Castro Leal 1969: 1178) telle est la définition proposée dans les années 1960 par Antonio Castro Leal à la fin de son anthologie sur la Novela de la Revolución ${ }^{4}$ dans les pages consacrées aux « mexicanismes » nés, écrit-il, « durant les années de la Révolution » (Ibid. : 1171). Mais si le mot appliqué à l'homme était apparu timidement durant les troubles révolutionnaires, à la fois dans les romans et les «corridos»-ces chansons à la gloire des révolutionnaires ou autres héros populaires - c'est surtout grâce au nationalisme des années 1930-1940 que le « macho » s'est imposé au point de devenir l'un des symboles de la nation post-révolutionnaire (Gutmann 2000 : 322-324 ; Machillot 2011).

Un processus qui, pour sa réussite, devait nécessairement passer par un média populaire sous la coupe de l'Etat : le cinéma (Arroyo Quiroz et al. 2011). Ce dernier remporta en effet très vite un énorme succès, présentant d'ailleurs dès les années 1930 l'Etat de Jalisco comme une région à la fois bucolique et idyllique, remplie de cavaliers courageux et séducteurs, de «charros » à la voix d'or.

Réinvention régionale de la nation donc, mais aussi réinterprétation du "macho » révolutionnaire pour des temps de paix (Machillot 2011). En effet, le « macho » de la « Epoca de oro $»^{5}$ du cinéma mexicain, s'il garde parfois un aspect guerrier dans des productions mettant en scène la Révolution, devient généralement un produit plus souriant et sympathique, séducteur enfin (Ibid.), et ce, lorsqu'il ne se transforme pas dans les années 1970 en dragueur impénitent et d'origine invariablement populaire.

4 « Roman de la Révolution », genre littéraire populaire englobant pratiquement toute production sur la Révolution.

5 L' « âge d'or » du cinéma mexicain, expression qui désigne la production cinématographique mexicaine comprise entre la fin des années 1930 et les années 1960. 
Bref, du début du $20^{\text {ème }}$ siècle à nos jours, l'image valorisante du « macho » évolue et se diversifie, tout en restant dans sa version régionale, en tant que « macho » de Jalisco, un symbole de la « mexicanité » (Machillot 2010 : 174-233).

Contrairement à ce que l'on pourrait croire, la caractérisation valorisante du « macho » n'a cependant jamais fait l'unanimité. En 1915, le roman Ceux d'en bas (2007) où a été observé l'un des premiers usages du mot (Gutmann 2000 : 320 ; Machillot 2011 ; Machillot 2010 : 174-175) dressait déjà un panorama très sombre de la Révolution et de ses héros. Le temps passant, ces attaques devinrent de plus en plus explicites tant de la part d'essayistes que d'universitaires ou de scientifiques, mexicains et américains, comme Ramos, Caso, Paz, Paredes, Díaz-Guerrero, Lewis, etc., que de romanciers de réputation internationale comme Juan Rulfo ou Carlos Fuentes (Machillot 2010 : 132-173 ; Machillot 2011).

Une critique qui, à ses débuts en particulier, ne s'exerçait pas tant contre une image dominatrice de la masculinité que contre un certain type de nationalisme (Machillot 2011)... La question des rapports entre l'homme et la femme n'apparaît de fait que peu à peu pour s'imposer définitivement dans les années 1970, grâce notamment aux luttes féministes et en faveur des minorités sexuelles (Machillot 2010 : 141-164 ; Machillot 2011) et à l'internationalisation de la cause des femmes ${ }^{6}$, au point que l'on confond maintenant le « macho » avec le « phallocrate », et le « machisme » avec la domination masculine. C'est ainsi qu'une informatrice, vidéaste proche de la trentaine, un soir d'août 2008, faisant référence à un de ses collègues de travail, parlait péjorativement de « très petit macho », dans le sens à la fois de chef autoritaire et dominateur et de « dragueur » se croyant « irrésistible $»^{7}$ et qu'une autre, jeune diplômée de licence de langue, qualifiait avec mépris en mars 2009 de « machito » - « petit macho » - le fiancé d'une de ses amies jugé trop possessif... Un contre-modèle donc, que condamne désormais l'Etat lui-même, comme en témoignent des observations recueillies par nos soins dans des ateliers offerts par des organismes gouvernementaux chargés de lutter contre la violence contre les femmes et de diffuser la perspective de genre, les Instituts des femmes - Institutos de las Mujeres -, ainsi que cet extrait du très officiel « Glossaire du genre »-Glosario de género (2007) - proposé par l'Institut national des femmes - INMujeres :

Machisme

Ensemble de croyances, conduites, attitudes et pratiques sociales qui justifient et promeuvent des attitudes discriminatoires contre les femmes ${ }^{8}$. (Glosario de género, 2007)

Il n'en demeure pas moins que la culpabilité des femmes, et en particulier des mères, dans la transmission et la reproduction du machisme est devenue à Guadalajara et plus généralement au Mexique un lieu commun. Elles feraient, littéralement, les

6 Le Mexique a signé au sein de l'ONU, en 1995, la plateforme d'action dite de Beijing, l'objectif étant de favoriser « la montée en puissance du rôle de la femme dans la société » - « la potenciación del papel de la mujer en la sociedad. » (Informe de la Cuarta Conferencia Mundial..., 1995, p. 8).

7 Soit : « muy machito », « un galán », « se cree irresistible ».

8 « Conjunto de creencias, conductas, actitudes y prácticas sociales que justifican y promueven actitudes discriminatorias contra las mujeres. » (Glosario de género, 2007) 
machos : «ce sont elles qui les font », " les mères sont les principales coupables ${ }^{9}$ nous confièrent ainsi des étudiantes de licence en mars 2009 dans une discussion sur le « machisme ». «Elles les élèvent comme ça »- «Ellas los educan así »- me commenta un jour de 2010 une militante féministe. Une accusation qui, tout en se voulant le plus souvent critique contre le machisme, en reproduit paradoxalement les stéréotypes lorsqu'elle délègue à la femme la responsabilité - entière et donc assumée par elle seule - de l'éducation.

Si le « machisme » est donc désormais officiellement condamné comme étant un mal « masculin », la femme n'en est donc pas moins désignée au quotidien comme l'une, si ce n'est la responsable de la reproduction de celui-ci. Un processus qui reproduisant la logique de ce qui est dénoncé tend par conséquent à culpabiliser la femme, voire à la stigmatiser... Et ce, doublement : en tant qu'élément passif au sein d'une relation de domination et en tant qu'agent " actif » dans le processus de reproduction du « machisme ».

Les définitions négatives du « machisme » et du « macho/machiste » n'épargnent donc pas complètement les femmes, néanmoins elles s'imposent - officiellement tout du moins - au sein d'un Etat qui autrefois défendait leur sens positif. Mais ce n'est pas là la seule contradiction. Le rejet actuel coexiste en effet, au quotidien, avec un usage encore valorisant du « macho ». Pourquoi ?

\section{Des différents usages valorisants du stéréotype au quotidien}

Il y a bien sûr, pour utiliser une expression d'Herzfeld, une « nostalgie culturelle » ou « folklorique » (2007 : 54) qui fera que, outre les rediffusions de films de l' « Epoque d'or » du cinéma mexicain et les rééditions régulières des « classiques » du Roman de la Révolution, toute une « imagerie » de la Révolution et de la post-révolution et des qualités supposées des hommes de cette époque sera cultivée par des chanteurs, par des acteurs, etc., au travers de productions artistiques mais aussi de manière plus quotidienne grâce à une « projection » de soi. Une mémoire des « machos » donc qui, tout en se perpétuant, reste liée à un Etat, Jalisco.

Lieu « historique » par excellence dans le sens où ses habitants y retrouvent « les traces diverses d'une implantation ancienne, le signe d'une filiation » (Augé 1994 : 156), l'Etat et ses habitants continuant en effet à être associés à la " mexicanité » et aux « machos ». Un Etat de Jalisco que le gouvernement régional promeut d'ailleurs sous la forme de «Jalisco es México », soit « Jalisco c'est le Mexique », slogan qui accompagne désormais toutes les manifestations artistiques et, plus particulièrement, folkloriques de la région.

Mais si la tradition et en particulier la revendication de la « mexicanité » peuvent servir à perpétuer des pratiques qui nuancent, voire contredisent, certaines politiques officielles, elle passe aussi par un discours qui n'est plus seulement une " nostalgie folklorique » connotée de nationalisme mais une véritable revendication d'un ethos mexicain et surtout masculin qui peuvent offrir, comme le souligne Herzfeld (2007 : 69) « un sentiment d'identification durable », et ce même et malgré le fait que ces

9 «Ellas los hacen », « las madres son las principales culpables ». 
« modèles patriarcaux » soient souvent « sources d'embarras » ( Ibid.) pour des secteurs plus progressistes et - officiellement tout du moins - pour l'Etat...

\section{« Parole de macho ", virilité et noblesse de l'homme}

« Palabra de macho », expression commune qui équivaut à notre « parole d'honneur ». « Homme », « honneur », « macho » : que les trois se confondent n'est pas un hasard. Comme Pitt-Rivers l'a montré pour l'Andalousie, s'il veut être vraiment un " homme », un individu doit être à même de démontrer son « honneur » en le défendant (1983) et être à même de le défendre, au Mexique, c'est être un «macho ».

La parole par ailleurs engage, littéralement, l'honneur. Une autorité qui se fonde sur l'action et sur l'exemple - pour Pitt-Rivers l'honneur peut aussi découler « d'une conduite exemplaire » (1983 : 38). Car la parole réputée rare de l'homme, parole dont la rareté en fait une parole qui compte à l'opposé du bavardage des femmes - dans Los Altos, région rurale à l'est de Jalisco, est admiré l' " homme de peu de mots mais qui valent beaucoup », " el hombre de pocas palabras pero que valen mucho »- est une parole qui est supposée s'accomplir... « Yo hago lo que digo »- « Moi, je fais ce que je dis »- entend-on ainsi à Guadalajara, contre ceux qui « ne font que parler » - « nada más hablan ». Un honneur qui oblige au respect de la parole, à l'adéquation entre ce qui est dit et ce qui est fait contrairement une fois encore à celui qui, hypocrite, exige de faire ce qu'il dit et non ce qu'il fait - « Haz lo que yo digo pero no lo que yo hago ». Un honneur qui se résume tout entier dans la virilité supposée de l'homme : sa force, son courage, sa masculinité opposée à la féminité de l'Autre - la femme, l'homosexuel.

De fait, comme le remarque Jesús Silva-Herzog Márquez, professeur du Instituto Tecnológico Autónomo de México et collaborateur régulier du journal Público, à propos de l'appel du sénateur du PRI, Beltrones, au président Calderón à « montrer ce qu'il a dans la culotte $»^{10}$ (Mercado 20 mars $\left.2009: 20\right):$ " la remise en question de la virilité de l'adversaire continue à être monnaie courante $» "$ (Silva-Herzog Márquez 23 mars 2009 : 16). Ces expressions figées, injonctions à " se comporter comme un homme »- «portarse como hombre» - à « agir comme des hommes » ou « comme des machos »- « actuar como hombres », « actuar como machos »-ou plus fréquemment encore à " avoir des couilles »" ${ }^{12}$ - littéralement des " œufs », " tener huevos » - sont non seulement communes, mais ont un effet performatif évident... C'est ainsi qu'un chef de chantier en septembre 2008, lançant un «Tiens le coup comme les machos !»« ¡Aguantate como los machos! - à un maçon qui se plaignait de l'instabilité d'une échelle, ne reçut aucune réponse, le jeune homme grimpant sans plus rien dire...

10 « Beltrones a Calderón : fájese los pantalones » (Mercado 20 mars 2009 : 20).

11 «Es notable, por ejemplo, que el cuestionamiento de la virilidad del adversario siga siendo moneda común. » (Silva-Herzog Márquez 23 mars 2009 : 16).

12 Pitt-Rivers observe un phénomène équivalent avec l'expression espagnole « cojones » (1983: 49). 
Toujours selon cette conception du «macho », les centaines de femmes ${ }^{13}$ assassinées à Juárez deviennent en 2004 pour le groupe de musique $«$ norteña $»{ }^{14}$ le plus populaire, Los Tigres del Norte, une « honte nationale ». Les crimes étant demeurés impunis, les hommes sont en effet accusés de ne pas assumer leurs responsabilités, donc d'avoir perdu «lo macho »-outre l'honneur national donc, la virilité :

Les mortes de Ciudad Juárez sont une honte nationale.

$[\ldots]$

On entend des commentaires honteux dans le monde entier

La réponse est très simple pour ceux qui connaissent la vérité

On a perdu notre virilité [se nos quitó lo macho] ou alors nous manquons de dignité $^{15}$.

Même si la domination masculine a quelque peu perdu de sa superbe, la croyance en des qualités nobles et proprement masculines qu'incarnerait la figure du « macho » n'a donc pas totalement disparu. C'est ainsi qu'en 2008, et ce, non sans nostalgie, Saúl nous confiait que ce que les habitants d'un village de Los Altos entendaient généralement par « macho » ce n'était pas forcément cet homme violent et impulsif qui vint selon lui avec la modernisation de la région dans les années 1970 mais l'homme de parole « hombre de palabra »-, sachant se faire respecter - « se hacian respetar » - tout en étant « droit » et « juste »- « cabal ». Homme d' « honneur», dont la « parole » est « de macho » qui se construit tout autant contre la féminité supposée de l'Autre...

\section{Le « macho » contre l'homosexuel}

«Afrancesado» : littéralement « francisé », l'expression sert d'abord à désigner ceux qui ont pris parti dans les guerres en faveur des envahisseurs, contre l'Espagne pendant les guerres napoléoniennes (Real Academia Española 1970 : 33), puis on le retrouve au Mexique lors de l'Intervention française de 1862-1867. De fait, l'historienne Erika Pani rapporte que le journal satirique La Orquesta se moquait souvent de « certains mannequins $»^{16}$ (Pani 2000 : 146). Des figures de l'élégant qui annoncent bien sûr celle du « catrín », ce dandy précieux de la fin du $19^{\text {ème }}$, les mignardises supposées des uns et des autres renvoyant enfin à la féminité.

13 Certaines ONG estimaient, entre 2008 et 2012, à plus de 900 le nombre de femmes assassinées ou portées disparues à Ciudad Juárez, dans le nord du pays (El Universal, 2012). La Cour interaméricaine des droits de l'homme évoquait, elle, le chiffre de 450 femmes sauvagement assassinées, souvent violées, entre 1993 et 2009 (Amnesty International 2009).

14 Musique originaire du nord de la république.

15 «Las muertas de Ciudad Juárez son vergüenza nacional//[...]// Vergonzosos comentarios se escuchan por todo el mundo/La respuesta es muy sencilla cuáles saben la verdad/Ya se nos quitó lo macho o nos falta dignidad. »

16 « [...] ciertos maniquís » journal La Orquesta de 1865 cité par Erika Pani (2000 : 146). 
Un « jeune gommeux », gentiment bêta ${ }^{17}$ (Ibid. : 146) converti en « Fleur de Paris $»^{18}$ (Ibid. : 147) auquel il est facile d'opposer la rudesse et la pilosité d'un Mexicain jugé authentique - exact contraire du " faux » mexicain xénophile - et sans complexe, coiffé de son " grand chapeau », vêtu de " pantalons/aux agrafes argentées » buvant du " pulque $»^{19}$ (Ibid. : 146). L'image, tout en renvoyant à celle des combattants des guerres d'indépendance, annonce le stéréotype non moins populaire dans le cinéma des années $1940 \mathrm{du}$ " macho » mexicain, rude, viril et grand buveur. Contre l'Autre efféminé donc, un soi viril. L'image perdurera...

C'est ainsi qu'entre 2007 et 2011, on entendait encore, lors de discussions de 《cantinas ${ }^{20}$ notamment, l'expression « macho » mais surtout sa dérivation « machín » lorsque se défendant d'allégation malicieuse contre leur sexualité, des clients - maçons, mécaniciens, voire petits commerçants - rétorquaient des « Moi ? Putain... Si je suis bien macho : j’adore les nanas !» - ¿ ¿Yo ? Uta... ;Si yo soy bien machín : me encantan las viejas! »- ou « Non! Pour rien au monde! Si moi je suis un vrai macho!»-« ¡No! ¡Qué va ! ¡Si yo soy bien machín!» - voire des superlatifs comme « Non. Comment peux-tu croire ça ? Ce mec-là est un super macho... »- «No. ¿Cómo crees? Este guey es un machototote... »-sous les rires ou les plaisanteries de leurs compagnons... Et ce, quand le nationalisme ne pointait pas au détour d'une phrase : « iSi nosotros, los mexicanos, somos bien machos ! " - Si nous, les Mexicains, nous sommes bien machos $! »^{21}$.

Nous ne pouvons ignorer, ici, ce que Beck ou Giddens désignent la « réflexivité » des acteurs (2008) vis-à-vis non seulement de leurs propres comportements mais de l'image et des discours que les autres peuvent se faire d'eux et de leur communauté. Comme le relève Herzfeld (2007 : 242), le recours au stéréotype peut alors devenir ironique. Au Mexique, on observa ainsi, occasionnellement, une «tomada de pelo », soit un « foutage de gueule », dont le chercheur fit même parfois les frais... et la revendication devient alors provocation. Mais plus souvent cette conscience s'exprime sur le mode du regret et le «nosotros los mexicanos somos bien machos » fait alors office de condamnation, voire d'appel implicite au changement...

Si l'ironie, voire le regret ne sont pas absents, l'homophobie cependant favorise la revendication de la figure du « macho » ou du « machín ». Et il n'est peut-être pas complètement déraisonnable, en effet, dans une société qui peut se montrer homophobe, de réaffirmer sa virilité, et ce, quitte à avoir des relations sexuelles avec d'autres hommes...

17 «Lechuguino [...] bicho-manso/que del mono tiene mucho/es nieto del aguilucho/ y primo hermano del ganso »/« Jeune gommeux [...] bestiole-bêta/qui a beaucoup du singe/petit-fils de l'aiglon/et cousin germain de l'oie » (Ibid. : 146).

18 «Flor de París » (Ibid. : 147).

19 « [mexicano vestido de] sombrerote [...] y calzoneras de plateados broches » (Ibid. : 146). « Pulque » : Alcool aux origines préhispaniques, tiré de la sève de l'agave, sirupeux et surtout très populaire.

20 Bars populaires autrefois interdits aux femmes.

21 Ce qui laisse supposer, de manière un peu provocante, que les autres ne le sont pas... 


\section{De la régulation des interactions ou comment être « macho " et « hétérosexuel » tout en ayant des relations sexuelles avec d'autres hommes?}

L'intimité culturelle, quand ce n'est pas l'observation de l'intimité elle-même, montre comme le souligne Herzfeld (2007) des réemplois pour le moins complexes et parfois inattendus des stéréotypes genrés et nationaux : stratégies, jeux avec et contre l'Etat mais aussi avec et contre la norme qui peuvent se révéler être par ailleurs, comme dans le cas que nous décrirons à présent, commerciaux. Illustration grâce aux résultats obtenus au travers de l'analyse de la presse gay et surtout de l'observation sur le terrain de relations entre des clients s'assumant comme homosexuels et des prostitués ou des amants se revendiquant, eux, comme hétérosexuels dans des estaminets du centre de Guadalajara, au Mexique, dans l'Etat de Jalisco entre septembre 2007 et août $2010^{22} \ldots$

De fait, premier point, comment être un " macho » et se considérer comme hétérosexuel tout en ayant des relations avec des homosexuels déclarés ? Il convient pour comprendre cela de préciser à la fois les circonstances et le contexte. Il existe au Mexique - comme le montre Córdova Plaza pour Veracruz (2005 : 228) - ce que les milieux gays nomment des « chacals "- «chacales »-, terme péjoratif à l'origine qui sert en fait à désigner un homme plutôt métissé, généralement musclé, d'aspect très masculin, qualifié de « macho » et souvent jugé dangereux par ceux qui le fréquentent - le mot « chacal » servant aussi au quotidien à désigner un « voyou »-, de statut social modeste et manuel de formation - d'après nos observations : maçon, peintre, déménageur, cireur de chaussures, etc. Généralement actif lors de relations homosexuelles, le « chacal » ne se reconnaît pas pour autant comme bisexuel ou gay et il est souvent confondu - en raison de rapports souvent "intéressés » - avec le « chichifo »-terme servant à désigner familièrement et péjorativement un « prostitué ».

Les «chacales » recourent de leur côté, pour se définir, à des expressions telles que « heterosexual », « hombre », « machín ${ }^{23}$. Un rôle auto-désigné de « macho » qui répond de fait à des stéréotypes en vogue dans les milieux homosexuels qui vont même jusqu'à décrire les « chichifos » et les « chacales» comme des « supermachos» comme en attestent, par exemple, ces panégyriques publiés dans différentes revues gays et affines :

Qui n'a pas rêvé d'un "chacal »? [...] homme rude et masculin, de préférence brun [de peau] ? [...] Je n'ai qu'un seul reproche : le fait que les "chacals » [...] n'acceptent pas leur homosexualité ou bisexualité ${ }^{24}$.

(Fernández Arenas 3 juillet 2008)

22 On peut consulter sur le thème de l'homosexualité et de la prostitution masculine au Mexique des auteurs comme : Córdova Plaza (2005), Carrier (2003), González Pérez (2003), Nuñez Noriega (2000 et 2007), Prieur (2008).

23 « Hétérosexuel », « homme », « macho ».

24 «Quién no ha soñado con un chacal? ¿[...] hombre de aspecto rudo y varonil, de preferencia moreno? [...] Sólo tengo un inconveniente : que los chacales [...] no aceptan su homosexualidad o bisexualidad» (Fernández Arenas 3 juillet 2008). 
Chacal [...] le sexe des bas-fonds avec un macho mexicain.

Post-scriptum : il existe des gens snobs qui ne voient pas d'un bon oeil les "chacals » parce qu'ils disent que leur style de vie est complètement contraire au glamour qui caractérise le milieu, à ce qui est gay. Ah, mon chéri ! Mais à quoi donc rêves-tu quand tu dors $?^{25}$

(Torres 17 mai 2007)

Significativement, classes sociales et masculinité se confondent : le fantasme du « supermacho » se construisant à la fois contre l'image efféminée du snob et du bourgeois et la féminité prêtée au " gay » assumé ou « évident» - « obvio ». Il n’y a là d'ailleurs rien de bien nouveau, l'image du « supermacho » prêtée aux "chichifos » « virils » et aux « chacales » s'inscrivant dans la tradition des héros de la Révolution : une figure populaire métissée et musclée, conditions sine qua non de l'homme nouveau révolutionnaire, opposée aux blancs bourgeois xénophiles... Et il y a de cela, bien sûr, même si les exploits guerriers sont ceux de la petite délinquance ou mieux, dans le cas de prostitués, de la prouesse sexuelle.

Et là encore, la transformation du stéréotype s'inspire de la tradition : de celles des bandits virils d'une part et du « macho » comme machine sexuelle des films des années 1970-1990 (Machillot 2011)... Avec une différence cependant, l'imagerie cinématographique de ces années faisaient l'apologie de l'homme « moyen », de ce qu'en France à la même époque on appelait le «beauf» et au Mexique le «naco » alors qu'en comparaison "chichifos » et «chacales» sont dépeints comme de véritables Adonis - la réalité étant parfois toute autre. Le culte de l'apparence est en effet passé par là et ce dont fantasment finalement les amateurs de " chacales », c'est de corps bodybuildés sans bodybuilding, façonnés par le travail, d'un maçon mexicain en mister monde... Et ce stéréotype de se construire une fois de plus contre celui du bourgeois cultivé, oisif et efféminé, « cosmopolite », amant du « glamour » ou plus grossièrement contre les stéréotypes dont sont eux-mêmes victimes les homosexuels : ceux de la « tante » ou du travesti...

Il se trouve d'ailleurs que les attributs qui se rattachent aux stéréotypes du " supermacho » sont non seulement espérés et attendus mais parfois exigés à tout « chichifo » très « viril » ou « chacal » dignes de ce nom ${ }^{26}$ comme en témoigne cette déclaration d'un de nos informateurs, Javier, en mars 2008 :

[Le « chacal »] doit avoir un certain type physique très masculin... [...] de plus il y a un rapport avec un certain comportement, une attitude, une attitude en concordance avec ce physique [très masculin], d'homme un peu animalesque [sic] avec des attitudes un peu brusques, brutales dans certains cas... ${ }^{27}$

25 « Chacal [...] sexo de arrabal con un macho mexicano. Posdata : Existe gente snob que no ve con buenos ojos a los chacales, pues dicen que su estilo de vida es totalmente contrario al glamour que caracteriza al “ambiente”, a lo gay. ¡¡Mi vidaaa!!! A qué le tiras cuando sueñas, mexicano » (Torres 17 mai 2007).

26 Goffman parle d' « attente normative » (1975:12).

27 «Tiene que tener un cierto tipo de físico que se vea muy varonil dentro de lo que es varonil... [...] además tiene que ver con un cierto comportamiento una actitud, una actitud en concordancia con ese fisico, de hombre un poco animalesco con actitudes un poco bruscas, brutales en algunos casos... » 
Le stéréotype sert donc à anticiper la rencontre, en prêtant à l'autre des traits et des caractéristiques que l'on s'attend d'autant plus à trouver que du désir s'en mêle... Dans La domination masculine, Pierre Bourdieu observe ainsi que la féminité n'est autre qu'une « forme de complaisance à l'égard des attentes masculines, réelles ou supposées » (Bourdieu 2002 : 94-95). Quant à nous, nous notons qu'il existe aussi une sorte de « complaisance » masculine tant à l'égard des attentes féminines que masculines.

Accommodation aux attentes des autres observée notamment au travers du spectacle de « chichifos » musclés, habillés en « cholos »-membres de bandes de quartier - ou en « rancheros »- « éleveurs », " paysans » coiffés de chapeaux et chaussés de santiags dans un bar traditionnel, une « cantina » du centre de Guadalajara, le Chivas, fréquentée aussi bien par un public de travestis et d'homosexuels que par des hétérosexuels de classes moyennes et défavorisées. Scène que nous pourrions évoquer de façon assez typique, à partir de nos observations sur le terrain, de cette façon :

Samedi 17 janvier 2009. Cantina Chivas. 21h-1h. Les « chichifos 》 discutent et boivent au comptoir, certains sont tournés vers la salle, la plupart ont entre vingt et trente ans. L'un d'eux, plus âgé, est grand et mince. Outre la moustache noire, il porte le costume du dimanche des "rancheros " : la chemise de cowboy ornée de chevaux, le chapeau de la couleur daim du pantalon, des bottes et un ceinturon avec un étalon sur la boucle... Les plus jeunes ont une apparence plus urbaine et plus agressive : Luis, 26 ans, est en marcel et montre des muscles gagnés, dit-il, sur le ring ; à une table voisine un jeune homme brun-jambes ouvertes, bien campé sur sa chaise, très musclé, cheveux rasés à la G.I., marcel noir, pantalon kaki, une vingtaine d'années et des lunettes de soleil - reste longtemps à boire, seul, ce qui ressemble à du rhum ou à du brandy avant d'être rejoint par un homme d'une cinquantaine d'années, cheveux blanc et maniéré. Un autre prostitué est habillé comme un "cholo», chemise à carreaux ouverte sur un tee-shirt blanc, pantalon extra large et tombant. Un "chichifo », chemise ouverte sur une chaîne avec une croix, cheveux courts et moustache très noirs est entré avec deux clients très efféminés dont l'un ne cesse de se lever pour parler en jouant avec une écharpe. Les " chichifos " restant se ressemblent : entre vingt et vingt-cinq ans, grands, ils déambulent dans la salle, sortent puis réapparaissent seuls ou par deux pour s'accouder au comptoir. L'un porte un sweet-shirt sombre avec une capuche baissée sur les yeux, mains dans les poches, regard fixe, il contemple la salle, il ne parle ni ne sourit... Les autres ressemblent à $n$ 'importe quels travailleurs manuels : jeans amples, chemises à manches retroussées ou simples tee-shirts sur les bras noués, chaînes avec des croix aux cous, cheveux plutôt courts, l'un porte une casquette avec le sigle d'une marque ou d'une équipe... Tous ont la peau plutôt brune...

Déguisements ? Même si s'habiller en « ranchero » en pleine nuit dans le centreville relève de la représentation, force est de reconnaître que celle-ci est, de l'avis de tous, convaincante et personne ne se risquerait à les confondre avec des simulateurs : la différence, affirment encore une fois les informateurs, c'est l'attitude. Pour notre part nous parlerions plutôt d'attributs : gestes brusques et maladroits, voix graves, vocabulaire familier qui tous correspondent aux canons traditionnels de la virilité...

En outre, là ou la représentation d'artistes pop comme Village People se limite à l'habillement, les acteurs de la vie réelle - tout en étant sur une scène - se doivent 
d'apprendre leur rôle dans sa totalité... Cela implique de s'inventer une biographie : Luis sera boxeur, aura été six années en prison... Non que l'histoire soit forcément fausse d'ailleurs, mais les faits qui nous sont racontés par ce prostitué « viril » s'ajustent si parfaitement à ce qu'on pourrait attendre d'un mauvais garçon qu'on le soupçonne d'en ignorer ou d'en cacher d'autres... Car de toute évidence les « chichifos » n'ignorent pas la fascination de leurs « clients » pour le délinquant - Alonso, homosexuel d'une vingtaine d'années nous dira ainsi que pour lui l'image du «chacal» ou du «chichifo » est précisément celle d'un « cabrón », d'un " salaud », d'un « dur »-qui bien sûr fait irrémédiablement penser au Querelle de Brest de Genet (1953) ou à Fassbinder (1978) dans son adaptation du premier, mais aussi aux analyses sociologiques de Cohen dans Delinquent Boys où il affirme que le délinquant " représente le mâle méchant », « la virilité sans entrave » (Cohen cité par Goffman 1974 : 223).

Ce qui compte d'ailleurs, c'est l'image publique qui justifie pour la préserver la négation, le secret, voire la violence... Ainsi, interrogé sur pourquoi selon lui les « chichifos » très « virils » nient presque toujours avoir été pénétrés lors de relations homoérotiques, la réponse de Javier en mars 2008, informateur clef qui les a fréquentés durant plus de trente années est extrêmement lucide :

Pour se prostituer [les « chichifos »] doivent jouer le rôle de l'homme, de l'homme dans le sens où c'est celui qui pénètre... même si ça lui plaît [d'être pénétré] il doit réprimer d'une certaine façon ce... ce goût... et il ne doit pas le dire, moins encore ouvertement parce qu'alors il peut perdre jusqu'à ses clients... parce que dans certains cas c'est une question de survie, parce que l'image de " machos » qu'ils ont, c'est ce qui leur permet aussi d'avoir des clients... ${ }^{28}$

Le jeu répond donc à une stratégie commerciale, l'offre s'adaptant ici à la demande comme le confirme ailleurs cette déclaration de Victor, strip-teaseur à Jalapa, rapportée par l'anthropologue Córdova Plaza :

Un strip-teaseur ne va pas te dire "moi je suis gay», parce que la virilité est ce qui est le plus en jeu, il doit manier une image de force, d'homme, de masculinité. Alors c'est clair que si on sort [sur scène] et qu'on a des mouvements efféminés, eh bien on ne plaît plus. Bien sûr, l'image de supermacho plaît aux gens gays et aussi aux nanas. Il faut donc soigner cette image... ${ }^{29}$ (2005 : 226)

Dans le cas des « chichifos » ou d'autres travailleurs sexuels, la conformité aux stéréotypes n'est donc pas passive, ni même mécanique mais répond de toute évidence à des intérêts et à des stratégies circonstanciées, non seulement identitaires mais aussi commerciaux. Phénomènes qui montrent qu'il existe une réinterprétation souvent

28 «Para prostituirse tienen que jugar el papel de hombre, de hombre en el sentido de que es el que penetra ... aunque le guste tiene que reprimir de alguna manera ese... ese gusto... y no lo tiene que decir, mucho menos lo tiene que decir abiertamente porque entonces se le puede incluso acabar hasta sus clientes... porque en algunos casos va de por medio su supervivencia, porque la imagen que tienen de machos es lo que les permite también tener clientes...»

29 «Un stripper no te va a decir “yo soy gay”, porque está más en juego la hombría, como que se debe manejar una imagen de fuerza, de hombre, de masculinidad. Entonces, obviamente si sale uno y tiene movimientos afeminados, pues ya no gustó. Claro que a la gente gay le gusta la imagen supermacho y a las chavas también les gusta. Entonces hay que cuidar esa imagen...» (Córdova Plaza 2005 : 226) 
lucide du « macho » qui déborde les sens et les manifestations traditionnelles du stéréotype tout en s'inspirant de et en composant avec ceux-ci, constituant ainsi face aux politiques officielles de singulières niches de résistances...

\section{Conclusion}

La norme qui ratifiait la supériorité masculine, dont le stéréotype valorisant du « macho » était l'expression, n'est plus dominante. Officiellement tout du moins, car s'il y a bien eu une institutionnalisation réelle de la " perspective de genre » et de la lutte contre le « machisme » à travers notamment la signature d'accords internationaux et la création d'Instituts des femmes chargés de faire appliquer ces politiques, la réalité - sociale, politique, etc. - contredit, voire dément ces bonnes intentions...

Nous avons dès lors, et pour le moins, des normes et des discours différents qui coexistent et luttent au sein d'une même société. Tandis que les Instituts des femmes tentent - officiellement encore - en tant qu'institutions gouvernementales d'instaurer un nouvel ordre ou pour le moins de corriger l'actuel, ailleurs le « macho » continue à faire « sens » au sein d'un imaginaire de la domination qui, lorsqu'il ne présente plus la virilité comme une noblesse nationale, s'appuie encore sur un puissant sentiment homophobe...

Un « sens » dont les acteurs semblent conscients et avec lequel - comme dans le cas de relations entre clients homosexuels et prostitués « virils 》- ils peuvent jouer ou au contraire se conformer lorsqu'il s'agit notamment de « régler » au quotidien des interactions réprouvées par la norme. Un usage des stéréotypes en action qui se révèle par conséquent moins « automatique » et " figé » qu'on ne le dit. On présente en effet souvent ces derniers comme $s$ 'ils parlaient à travers l'acteur. Le stéréotype spontané et incontrôlable s'exprimerait malgré celui-ci ${ }^{30}$ - et à travers le stéréotype, la société, l'imaginaire, la mémoire collective... L'acteur inconscient serait en somme soumis au langage, prisonnier du symbolique, ventriloque d'un collectif... Ces images sont tentantes mais la voix des informateurs, l'analyse des interactions in situ, en somme l'emploi de l'ethnographie pour l'analyse de l'objet stéréotype nous montrent cependant qu'elles ne sont pas complètement vraies... Et c'est là, peut-être, outre la dynamique des acteurs et des cultures, l'une des leçons que pourrait nous donner l'anthropologie, comparée à d'autres disciplines, comme la psychologie sociale, qui se sont traditionnellement intéressées aux stéréotypes.

\section{Références citées}

Amnesty International, 28 avril 2009. « La Cour interaméricaine des droits de l'homme examine une importante affaire de violence contre les femmes au Mexique ». Disponible sur Internet [réf. du 29 mai 2010] : http://www.amnesty.org/en/library/asset/AMR41/023/2009/en/3e41b5ac-d0ff-4de7b7b7-b9b5034a58a7/amr410232009fra.pdf

Arroyo Quiroz, Claudia, James Ramey et Michael Schuessler (coord.), 2011. México imaginado. Nuevos enfoques sobre el cine (trans)nacional. Mexico : UAM.

30 Seca définit ainsi les stéréotypes comme « incitant les sujets sous leur emprise à des jugements hâtifs » (2005: 177). 
Augé, Marc, 1994. Pour une anthropologie des mondes contemporains. Paris : Flammarion.

Azuela, Mariano, 2007. Ceux d'en bas. Paris : Les Fondeurs de briques.

Beck, Ulrich, Anthony Giddens et Scott Lash, 2008. Modernización reflexiva : politica, tradición y estética en el orden social moderno. Madrid : Alianza Editorial.

Bourdieu, Pierre, 2000. Esquisse d'une théorie de la pratique précédé de trois études d'ethnologie kabyle. Paris : Seuil.

-, 2002. La domination masculine. Paris : Editions du Seuil.

Carrier, Joseph, 2003. De los otros. Intimidad y homosexualidad del occidente y el noroeste de Mexico. Mexico : Editorial Pandora.

Castro Leal, Antonio (coord.), 1967. La novela de la Revolución mexicana, t.I et II. Mexico : Ediciones Aguilar.

Córdova Plaza, Rosío, 2005. «Vida en los márgenes : la experiencia corporal como anclaje identitario entre sexoservidores de la ciudad de Xalapa, Veracruz », Cuicuilco 12 (34) : 217-218.

El Universal, 22 février 2012. « ONG denuncia 745 feminicidios en Juárez ». Disponible sur internet [réf. du 2 mars 2012] : http://www.eluniversal.com.mx/notas/831815.html

Fernández Arenas, Claudia, 3 juillet 2008. « iiiQue vivan los chacales ! ! », Anodis. Disponible sur Internet [réf. du 25 septembre 2008] : http://anodis.com/nota/11707.asp

Genet, Jean, 1953. Querelle de Brest. Paris : Gallimard.

Glosario de género, 2007, Mexico, Instituto Nacional de las Mujeres. Disponible sur Internet [réf. du 20 juin 2008] : http://institutodelasmujeres.jalisco.gob.mx/images/proyecto.swf

Goffman, Erving, 1974. Les rites d'interaction. Paris : Editions de Minuit.

-, 1975. Stigmate. Les usages sociaux des handicaps. Paris : Editions de Minuit.

González Pérez, César O., 2003. Travestidos al desnudo : homosexualidad, identidades y luchas territoriales en Colima. Mexico : Centro de Investigaciones y estudios Superiores en Antropología Social.

Gutmann, Matthew, 2000. Ser hombre de verdad en la ciudad de Mexico. Ni macho ni mandilón. Mexico : El Colegio de Mexico.

Herzfeld, Michael, 2007. L'intimité culturelle. Poétique sociale dans l'Etat nation. Laval : Presses Université Laval.

Informe de la Cuarta Conferencia Mundial sobre la Mujer Beijing, 4 a 15 de septiembre de 1995, 1995, ONU. Disponible sur Internet [réf. du 17 avril 2008] :http://www.eclac.org/mujer/publicaciones/ sinsigla/xml/3/6193/Plataforma.pdf

Instituto Mexicano de la Juventud, 2004. « Hablemos de masculinidades ». Disponible sur Internet [réf. du 26 décembre 2008] : http://ver2.imjuventud.gob.mx/masculinidades_contenido.asp

Machillot, Didier, 2007. « La polémique de 1925 : genre, champ littéraire et contraintes idéologiques dans "El afeminamiento en la literatura mexicana" » de Julio Jiménez Rueda, CRIMIC, Université Paris IV-Sorbonne.

-, 2010. «Machos » et « machistes ». Anthropologie de stéréotypes mexicains depuis l'Etat de Jalisco. Thèse soutenue sous la direction de Francis Dupuy. Université de Poitiers.

-, 2011. «"Machos" et "machistes" : (brève) histoire de stéréotypes mexicains », Amerika 4, disponible sur Internet [réf. du 27 octobre 2011] : http://amerika.revues.org/2149

Mellado, Pedro, 2008. « Mujer de mano pesada », Metro. La voz de Jalisco, 3 novembre $2008: 2$.

Mendoza, Vicente T., 1962. «El machismo en México al través de las canciones, corridos y cantares », Cuadernos del Instituto de Antropología, III : 75-86.

Mercado, Angélica, 20 mars 2009. « Beltrones a Calderón : fájese los pantalones », Público : 20. 
Monsiváis, Carlos, 2004. Escenas de pudor y liviandad. Mexico : Debolsillo.

Nuñez Noriega, Guillermo, 2000. Sexo entre varones. Poder y resistencia en el campo sexual. Mexico : Centro de Investigaciones y estudios Superiores en Antropología Social.

-, 2007. Masculinidad e intimidad ; identidad, sexualidad y sida. Mexico : UNAM-Programa Universitario de Estudios de Género.

Pani Erika, 2000. « Novia de Republicanos, franceses y emperadores : la ciudad de Mexico durante la Intervención francesa », Relaciones XXI (84).

Paredes, Américo, 1967. « Estados Unidos, México y el machismo », Journal of Inter-American Studies 9 : 65-84.

Pereira, Armando, 2004. Diccionario de literatura mexicana : siglo XX. Mexico : UNAM.

Pitt-Rivers, Julian, 1983. Anthropologie de l'honneur. La mésaventure de Sichem. Paris : Le Sycomore.

Prieur, Annick, 2008. La casa de Mema. Travestis, locas y machos. Mexico : UNAM-PUEG.

Real Academia Española. Diccionario de la lengua española, 1970. Madrid : Editorial Espasa-Calpe.

Seca, Jean-Marie, 2001. Les représentations sociales. Paris : Editions Armand Colin.

Silva-Herzog Márquez, Jesús, 2009. « El condimento del insulto », Público 23 mars 2009 : 16.

Torres, Alberto, 17 mai 2007. « Estereotipos de los chicos gay », Reportajes Metropolitanos. Disponible sur Internet [réf. du 25 août 2008] :http://www.reportajesmetropolitanos.com.mx/jovenes_mayo_07.html 
